\title{
Effects of salvianolate on myocardial perfusion after primary percutaneous catheter intervention in patients with ST-segment elevation myocardial infarction: a multicenter, randomized, double-blind, placebo-controlled study
}

\author{
Li Shen ${ }^{1,2 \#}$, Rende $\mathrm{Xu}^{1,2 \#}$, Jiasheng Yin ${ }^{1,2}$, Hao Lu ${ }^{1,2}$, Shuning Zhang ${ }^{1,2}$, Zheyong Huang ${ }^{1,2}$, Dong Huang ${ }^{1,2}$, \\ Hongying Liu ${ }^{2}$, Juying Qian ${ }^{1,2}$, Junbo $\mathrm{Ge}^{1,2}$; on behalf of the STEMI investigators group \\ ${ }^{1}$ Department of Cardiology, Zhongshan Hospital, Fudan University, Shanghai, China; ${ }^{2}$ Shanghai Institute of Cardiovascular Diseases, Shanghai, \\ China \\ Contributions: (I) Conception and design: J Ge, J Qian; (II) Administrative support: H Lu, S Zhang, Z Huang, D Huang; (III) Provision of study \\ materials or patients: J Yin, H Liu; (IV) Collection and assembly of data: L Shen, R Xu; (V) Data analysis and interpretation: L Shen, R Xu, J Yin; (VI) \\ Manuscript writing: All authors; (VII) Final approval of manuscript: All authors. \\ \#These authors contributed equally to this work. \\ Correspondence to: Junbo Ge. Department of Cardiology, Zhongshan Hospital, Fudan University, 180 Fenglin Road, Shanghai, China. \\ Email: jbge@zs-hospital.sh.cn.
}

Background: Despite prompt revascularization following acute myocardial infarction, poor myocardial perfusion commonly occurs due to impaired microvascular circulation and is an independent predictor of adverse outcomes. The current trial sought to examine the effects of salvianolate on myocardial perfusion in patients with ST-segment-elevation myocardial infarction (STEMI) who were undergoing primary percutaneous coronary intervention (PCI).

Methods: This randomized, double-blind, placebo-controlled, multicenter study evaluated the effects of intravenous salvianolate on the achievement of complete epicardial and myocardial reperfusion after PCI, defined as thrombolysis in myocardial infarction flow grade 3 and thrombolysis in myocardial infarction myocardial perfusion grade 3. We also measured plasma total creatine kinase-mass band fraction (CK$\mathrm{MB}$ )-estimated infarct size and echocardiography-derived left ventricular ejection fraction and recorded the 30-day clinical and safety outcomes. A total of 536 patients presenting with acute STEMI were randomized to receive either an i.v. infusion of salvianolate $(n=265)$ or placebo $(n=271)$.

Results: Salvianolate administration exerted beneficial effects on coronary microcirculation. There was a trend of reduced myocardial infarct size in the salvianolate group compared to the placebo group $(\mathrm{P}=0.070)$, although no significant difference in left ventricular ejection fraction was found between the two groups.

Conclusions: Salvianolate administration is associated with improved myocardial perfusion in patients with STEMI undergoing PCI. A larger study is required to assess the impact of this therapy on clinical cardiac outcomes.

Keywords: Salvianolate; myocardial infarction; primary percutaneous; coronary intervention; myocardial perfusion

Submitted Aug 12, 2020. Accepted for publication Sep 14, 2020.

doi: $10.21037 /$ atm-20-6081

View this article at: http://dx.doi.org/10.21037/atm-20-6081 


\section{Introduction}

Immediate reperfusion of ischemic with viable myocardium is necessary to reduce the amount of infarcted tissue and is the major therapeutic goal for patients with ST-segment elevation acute myocardial infarction (STEMI) $(1,2)$. With the development of therapeutic drugs, such as antiplatelet therapy, statins, $\beta$-blockade and angiotensin-converting enzyme inhibitors, and the progress of the treatment, especially the introduction of primary percutaneous coronary intervention (PCI), which can restore epicardial blood flow in the culprit coronary artery in a timely manner, the overall prognosis of STEMI patients has been dramatically improved (3). However, in a significant portion of patients, adequate tissue perfusion at the level of the myocardium still cannot be achieved, even after successful primary PCI (4). Poor myocardial perfusion despite the patency of the coronary artery, known as a "no-reflow" phenomenon and mainly attributed to microvascular injury, is associated with a higher occurrence of extensive infarction, postinfarction left ventricular remodeling, and higher mortality than good myocardial perfusion $(5,6)$. Although several strategies have been attempted to reduce inadequate myocardial perfusion in STEMI management, no therapeutic strategy for STEMI prevention or treatment has shown definitive efficacy in large randomized trials (7-9). Moreover, most of these pharmacological agents are typically utilized during or after PCI, well after no-reflow has occurred.

The dried root of Salvia miltiorrbiza (Danshen in Chinese) is one of the most commonly used herbal traditional Chinese medicines for cardiovascular indications. Salvianolate, a highly purified aqueous extract from Danshen, contains mainly magnesium lithospermate B $(\geq 85 \%)$, rosmarinic acid $(\geq 10.1 \%)$, and lithospermic acid $(\geq 1.9 \%$ ) (10). Salvianolate injection was approved by the China Food and Drug Administration for the treatment of stable coronary artery disease in 2005 . Salvianolate has been reported to exert cardiovascular benefits through diverse mechanisms that include scavenging oxygen-free radicals, inhibiting inflammatory response, and possessing antiischemic properties $(11,12)$. Furthermore, salvianolate has been applied in clinic as an adjuvant therapy to treat acute kidney injury and myocardial infarction in China $(11,13)$. In animal and experimental models, salvianolate has been shown to ameliorate microvascular injury and improve microcirculation after myocardial infarction (14). However, to date, limited data are available about the effect of salvianolate on microvascular flow and myocardial perfusion in STEMI patients. The present study was designed to assess the effect of salvianolate in STEMI patients treated with primary PCI. We hypothesized that perioperative administration of salvianolate may improve myocardial reperfusion and reduce infarct size compared with the standard procedure. We present the following article in accordance with the CONSORT reporting checklist (available at http://dx.doi.org/10.21037/atm-20-6081).

\section{Methods}

\section{Trial design}

The study was designed as a multicenter, double-blind, randomized, placebo-controlled trial to evaluate the effect of salvianolate injection on myocardial perfusion after primary $\mathrm{PCI}$ in patients presenting with acute STEMI. The study was registered (No. NCT03045562) and overseen by trial steering and data monitoring committees.

\section{Participants and eligibility criteria}

Eligible patients were those aged $\geq 18$ years and presenting within 12 hours of the onset of chest pain with electrocardiogram (ECG) features of acute STEMI ( $\geq 2 \mathrm{~mm}$ in 2 contiguous precordial leads or $\geq 1 \mathrm{~mm}$ in two peripheral leads) for whom the decision had been made to proceed with primary PCI of the occluded culprit artery.

The exclusion criteria were (I) patients with an allergy to salvianolate; (II) patients with mechanical complications; (III) patients with a history of severe renal or hepatic insufficiency; (IV) pregnant or breastfeeding women; and (V) patients who had difficulty in follow-up or communication or who had poor compliance. The study protocol was approved by the local ethics committee, and informed consent was obtained from all patients. All procedures performed in this study involving human participants were in accordance with the Declaration of Helsinki (as revised in 2013).

\section{Randomization and study procedures}

Patients who satisfied the inclusion and exclusion criteria were randomly allocated in a 1:1 ratio to the salvianolate and control groups through stratified randomization using a random number table in each study center by an investigator not clinically involved in our study. All patients received 
a loading dose of aspirin and clopidogrel according to the standard guidelines for STEMI in the emergency room (15). Unfractionated heparin $(70-100 \mathrm{U} / \mathrm{kg})$ was used as an anticoagulant agent during the invasive procedure. Salvianolate or placebo treatment was started immediately after the patient arrived at the catheter laboratory. The salvianolate and placebo were in identical in appearance and prepacked in sequentially numbered containers according to the randomization list. Patients in the salvianolate group received intravenous salvianolate $(200 \mathrm{mg}+100 \mathrm{~mL}$ of $0.9 \%$ saline at a rate of $100 \mathrm{~mL} / \mathrm{h})$, followed by continuous infusion $(200 \mathrm{mg}+$ $100 \mathrm{~mL}$ of $0.9 \%$ saline at a rate of $100 \mathrm{~mL} / \mathrm{h}$ per day) for 7 days. In the control group, the patients received $100 \mathrm{~mL}$ of $0.9 \%$ saline (placebo). Technical aspects of the primary PCI procedure and the use of glycoprotein IIb/IIIa inhibitors were left determined at the surgeons' discretion, and all the patients received optimal pharmacological therapy in accordance with current guidelines. Patients were asked to complete follow-up interviews at the outpatient department at 1 month. All adverse clinical events were recorded during both the hospitalization and follow-up periods.

\section{Study endpoints}

The primary endpoint of this study was the achievement of complete epicardial and myocardial reperfusion after PCI, defined as thrombolysis in myocardial infarction (TIMI) flow grade (TFG) 3, and TIMI myocardial perfusion grade (TMPG) 3. The secondary endpoints included corrected TIMI frame count (CTFC) and TIMI myocardial perfusion frame count (TMPFC) after PCI, complete ( $\geq 70 \%)$ STsegment resolution (STR) at 90 minutes post-PCI, and plasma total creatine-kinase mass band fraction (CK-MB) area under the curve (AUC).

\section{Angiographic analysis}

Angiographic assessment was performed at an independent angiographic core laboratory by experienced readers who were blinded to the clinical variables. The assessment included TFG and CTFC before and after the procedure, TMPG, and TMPFC. All analyses were carried out according to standard methods. TMPFC was calculated based on the final angiography after PCI and defined as angiographic frame numbers between myocardial blush appearing and disappearing distal to infarction-related lesion (16).

\section{Electrocardiographic (ECG) analysis}

ECGs obtained at admission and 90-min postprocedure (Figure S1) were analyzed as pairs at the ECG core laboratory by independent observers who were blinded to the clinical and angiographic data. STR was evaluated using standardized techniques and categorized as complete (>70\%), partial (30\% to $70 \%)$, or none $(<30 \%)$.

\section{Echocardiography and analysis}

A conventional echocardiogram was performed 7 days after angioplasty in the hospital and repeated at 30 days postprocedure. The left ventricular ejection fraction (LVEF) was estimated using the biplane Simpson method. In addition, myocardial contrast echocardiography (MCE) was also achieved at 7 days after primary PCI for quantitative assessment myocardial perfusion. According to the 17 ventriculus sinister segment piecewise method, myocardial contrast score of the segments were assessed to calculate the contrast score index (CSI) (17). All measurements were made by an independent experienced echocardiographic reviewer who was blinded to treatment assignment at the time of these measurements.

\section{Enzymatic infarct size}

Infarct size was estimated by measuring enzyme activity using CK-MB as a reference. The enzymatic activity was reported as IU/L and was assessed at the admission, and 6, 12, 24, and 36 hours after admission. The $0-36$ hour AUC (in arbitrary units) of the release of serum CK-MB was approximated in each patient using the trapezoid method as a surrogate marker of infarct size.

\section{Clinical and safety outcomes}

Clinical follow-up was performed at 30 days. Information on adverse events was obtained by a clinical review of the patients. The clinical outcome measures are reported as the composite of major adverse cardiovascular events (MACEs): all-cause death, reinfarction, and target vessel revascularization for ischemia and stroke. The frequency of adverse events (AEs), serious adverse events (SAEs) and patient discontinuations due to AEs are reported as safety outcomes. 


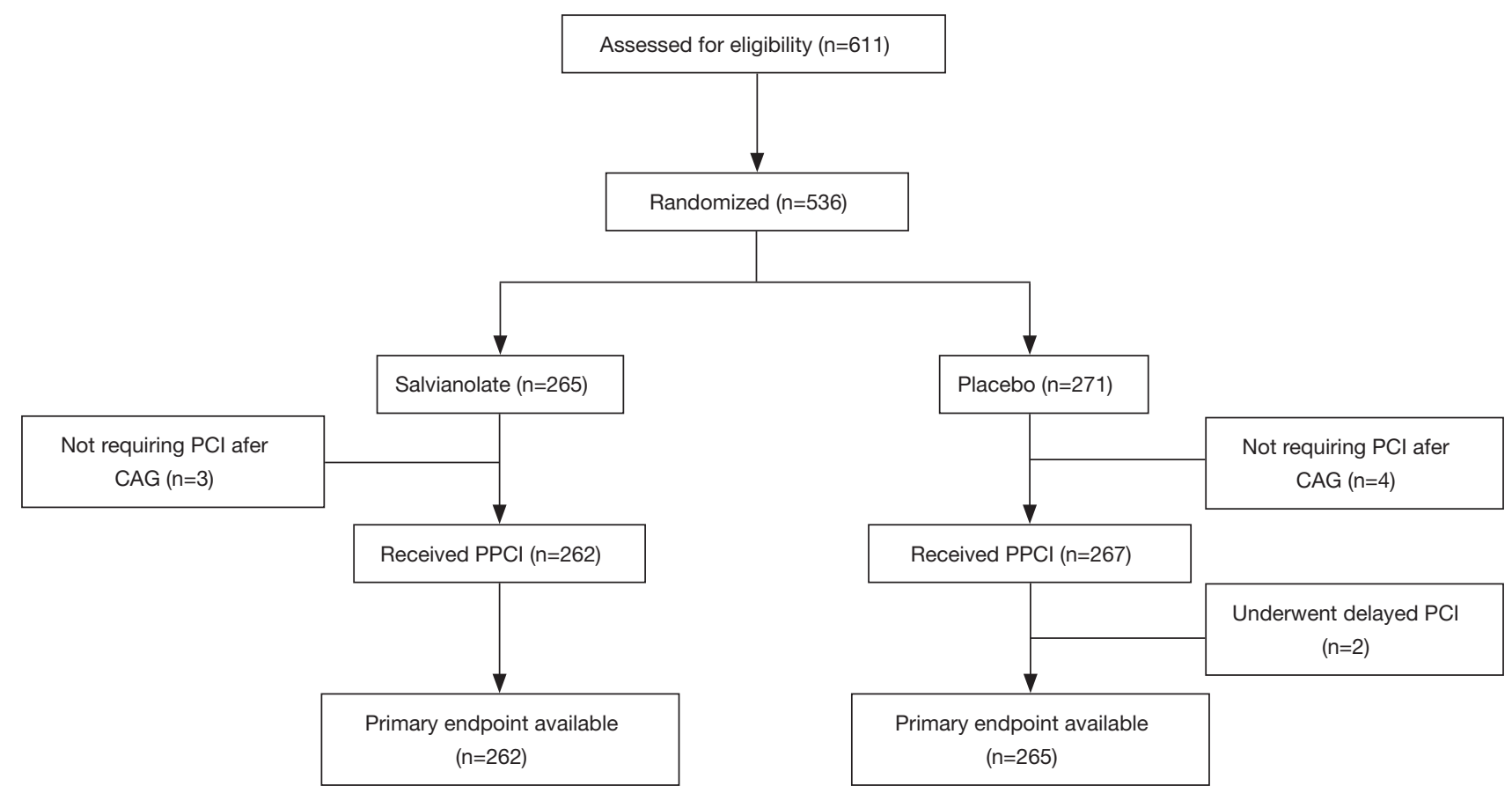

Figure 1 Flow chart of the trial. CAG, coronary angiogram; PCI, percutaneous coronary intervention.

\section{Sample size calculation}

Calculation of the sample size was performed based on previous studies. According to the data from the OnTIME 2 trail (18), we assumed the incidence of the primary endpoint (complete epicardial and myocardial reperfusion after primary PCI) in the placebo group would be $36 \%$ and that a relevant treatment effect would reduce this by $12 \%$ in the tested group. Therefore, given a type I error rate of alpha $=0.05$ and a power of $80 \%$ (type II error rate of beta $=0.2$ ), 214 patients would be required in each group. Considering a $20 \%$ drop-out or loss to follow-up, we planned to enroll a total of 536 patients.

\section{Statistical analysis}

The qualitative data are presented as numbers and percentages and were tested by Pearson's chi-square, Fisher's exact test, or the Cochran-Mantel-Haenszel test. The quantitative data are reported as the mean \pm standard deviation (SD) or medians with interquartile ranges, and were tested by Student's $t$-test or Wilcoxon's signed-rank test. A 2-sided alpha level of 0.05 was considered to indicate statistical significance, and $\mathrm{P}<0.05$ was defined as significant. Statistical analyses were performed with SAS software (SAS
Institute Inc., Cary, NC, USA), version 9.3. The figures were generated with Prism 6.

\section{Results}

\section{Patient characteristics}

From October 2016 to March 2018, a total of 536 patients with STEMI underwent randomization at 27 centers in China; the flow diagram of patients enrolled in the study is shown in Figure 1. There were 9 of 536 postrandomization exclusions for not requiring PCI $(\mathrm{n}=7)$ or symptomto-balloon time $>12$ hours $(n=2)$. The baseline patient characteristics are shown in Table 1. The distributions of the demographic and clinical characteristics were well balanced between the salvianolate and placebo groups. The mean age of the total population was 61.8 years. More than $80 \%$ of patients were males in both groups. There were no differences between the two groups in the prevalence of hypertension, diabetes, or hyperlipidemia. Both groups had no differences in blood pressure or heart rate, indicating a similar hemodynamic status.

Angiographic findings and procedural data are displayed in Table 2. The distribution of the infarct-related artery and preprocedural TIMI flow rates was not different 
Table 1 Baseline characteristics of patients

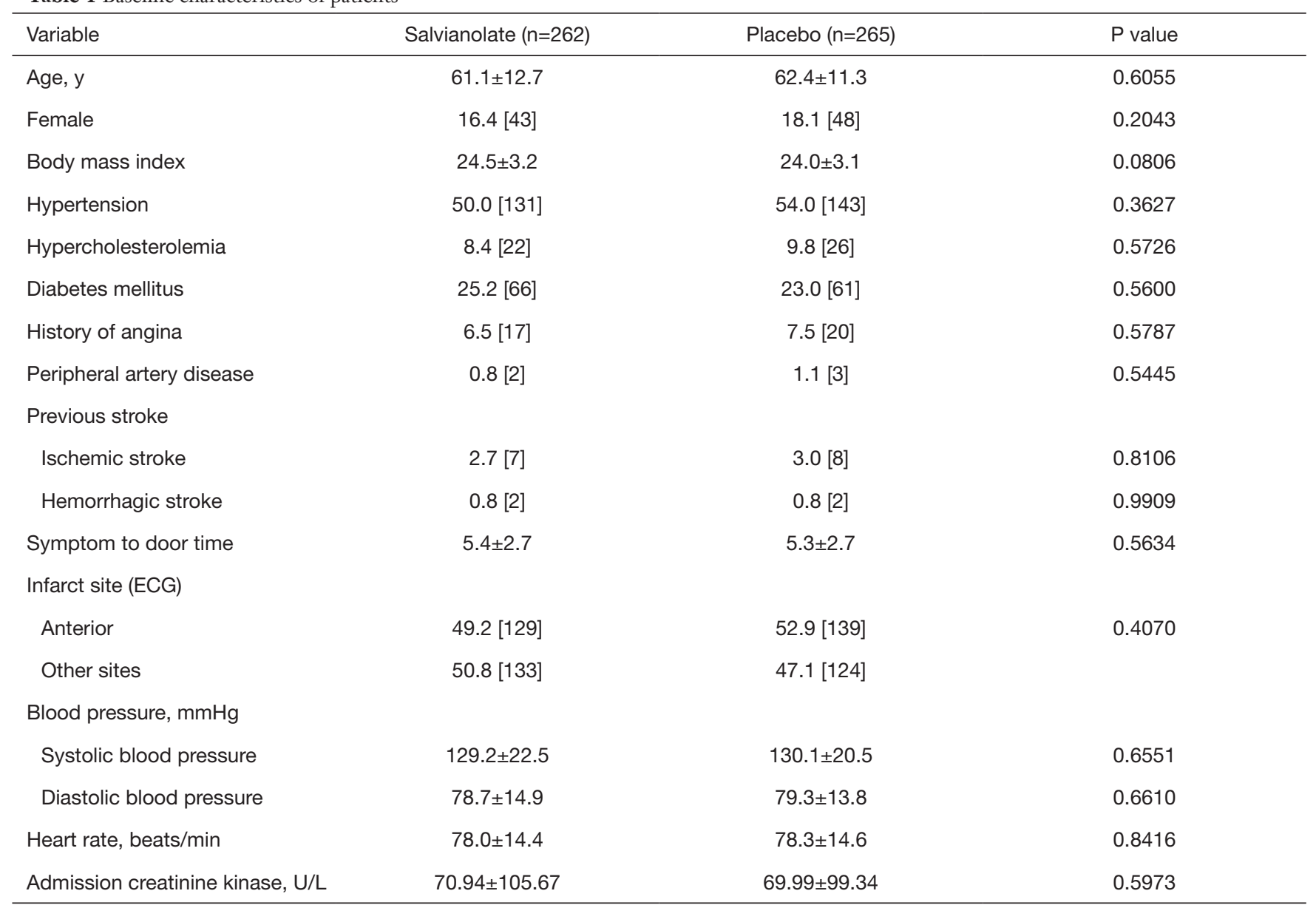

ECG, electrocardiogram.

between the two groups. Most patients (92.4\%) underwent stent implantation in the culprit coronary artery. The two groups were also similar with regard to the use of thrombus aspiration, glycoprotein IIb/IIIa inhibitors, sodium nitroprusside, and diltiazem during primary PCI. The rates of procedural complications, including coronary perforation, dissections, acute in-stent thrombosis, and major side-branch occlusion, were comparable between the two groups.

\section{Epicardial and myocardial reperfusion}

The rate of postprocedural TIMI 3 flow was approximately $90 \%$ in both the salvianolate and placebo groups. Although the incidence of normal epicardial flow (TFG 3) did not differ between the salvianolate and placebo groups $(91.6 \%$ vs. $88.3 \%, \mathrm{P}=0.208$ ) following $\mathrm{PCI}$, the occurrence of post-
PCI TMPG 3 was significantly higher among patients treated with salvianolate $(74.0 \%$ vs. $65.3 \%, \mathrm{P}=0.029)$. In addition, myocardial perfusion as assessed by complete STR was also obviously improved by salvianolate treatment (46.6\% vs. $37.7 \%, \mathrm{P}=0.047$ ). Further analysis showed that full reperfusion (primary end point, the presence of both TFG 3 and TMPG 3) following angioplasty was more common in the salvianolate group than in the placebo group (Figure 2). Furthermore, compared to the placebo group, salvianolate treatment resulted in a higher frequency of complete epicardial and myocardial reperfusion in all prespecified subgroups (Figure 3).

In addition to TFG and TMPG, the angiographic epicardial and myocardial flow was also evaluated by CTFC and TMPFC as continuous variables. Consistently, compared with those in the placebo group, patients in the salvianolate group had significantly better TMPFC, but 
Table 2 Angiographic characteristics

\begin{tabular}{|c|c|c|c|}
\hline Characteristics & Salvianolate $(\mathrm{n}=262)$ & Placebo $(n=265)$ & $P$ value \\
\hline \multicolumn{4}{|l|}{ Culprit vessel, n (\%) } \\
\hline LAD & $135(51.5)$ & $137(51.7)$ & \multirow[t]{3}{*}{0.796} \\
\hline RCA, dominant or balanced & $97(37.0)$ & $98(37.0)$ & \\
\hline LM & $1(0.4)$ & $0(0)$ & \\
\hline Multivessel disease, n (\%) & $40(15.3)$ & $45(17.0)$ & 0.593 \\
\hline Stent insertion, n (\%) & $244(94.2)$ & $243(92.0)$ & 0.3286 \\
\hline \multicolumn{4}{|l|}{ TIMI flow pre-PCI, n (\%) } \\
\hline 2 & $24(9.2)$ & $20(7.5)$ & 0.5033 \\
\hline 3 & $41(15.6)$ & $42(15.8)$ & 0.9497 \\
\hline \multicolumn{4}{|l|}{ TMPG flow pre-PCI, n (\%) } \\
\hline 0 & $184(70.2)$ & $189(71.3)$ & 0.7829 \\
\hline 1 & $31(11.8)$ & $22(8.3)$ & 0.1779 \\
\hline 2 & $11(4.2)$ & $10(3.8)$ & 0.8031 \\
\hline 3 & $36(13.7)$ & $44(16.6)$ & 0.3597 \\
\hline Thrombus aspiration, n (\%) & $90(35.2)$ & $86(32.8)$ & 0.5753 \\
\hline \multicolumn{4}{|l|}{ GPI, n (\%) } \\
\hline Sodium nitroprusside & $20(7.7)$ & $23(8.7)$ & 0.6802 \\
\hline \multicolumn{4}{|l|}{ Procedural complications, $\mathrm{n}(\%)$} \\
\hline Coronary perforation & $1(0.4)$ & $0(0)$ & 0.3141 \\
\hline Dissections & $2(0.8)$ & $3(1.1)$ & 0.6624 \\
\hline Acute in-stent thrombosis & $1(0.4)$ & $1(0.4)$ & 0.9936 \\
\hline Major side-branch occlusion & $3(1.2)$ & $3(1.1)$ & 0.9888 \\
\hline
\end{tabular}

LAD, left anterior descending artery; LCx, left circumflex artery; LM, left main; RCA, right coronary artery; TIMI, thrombolysis in myocardial infarction; PCI, percutaneous coronary intervention; TMPG, TIMI myocardial perfusion grade; GPI, GP IIb/Illa inhibitor. 

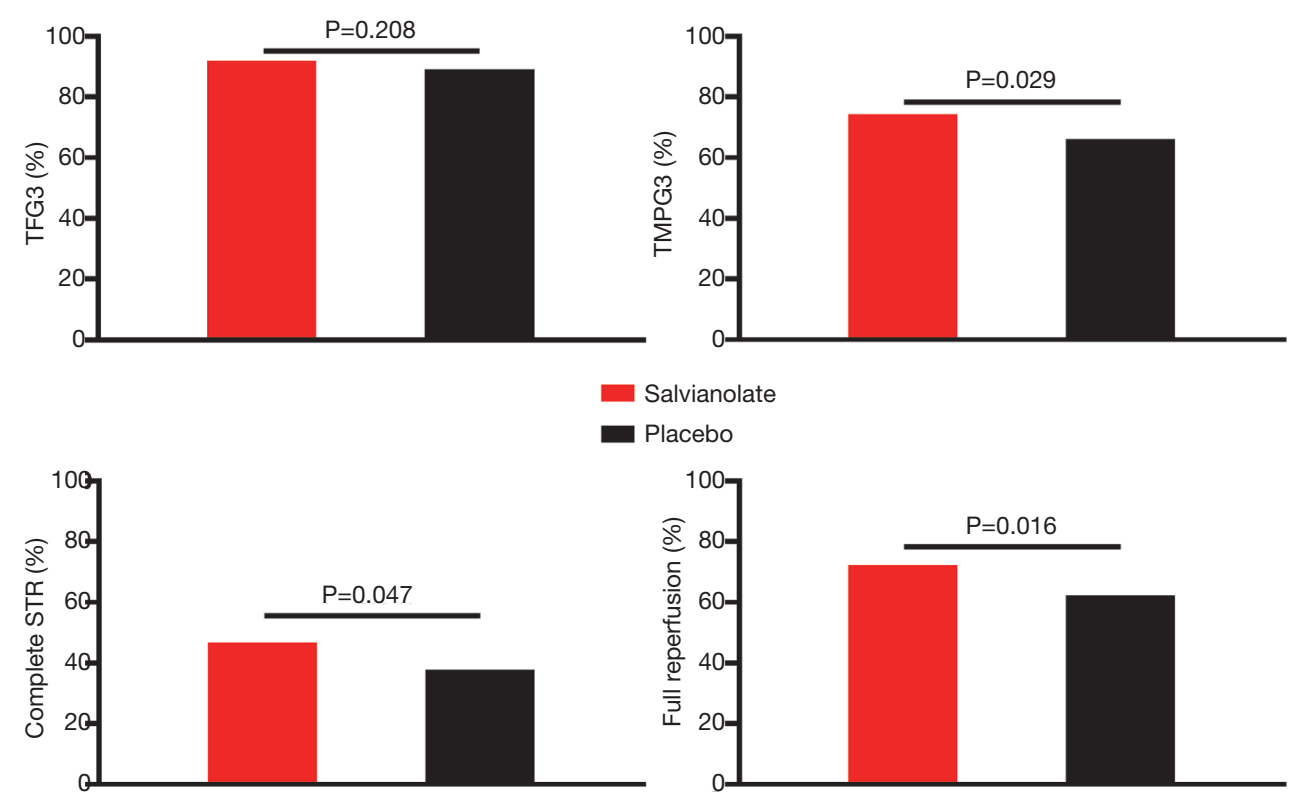

Figure 2 Prevalence of TFG 3, TMPG 3, complete STR, and full reperfusion after coronary intervention in the salvianolate group (n=222) compared with the placebo group ( $\mathrm{n}=221)$. Full reperfusion denotes the prevalence of patients who achieved TFG 3 and TMPG 3 following percutaneous coronary intervention. Statistical significance was determined with the Pearson's chi-square test. TFG, TIMI flow grade; TMPG, TIMI myocardial perfusion grade; STR, ST-segment resolution.

\begin{tabular}{|c|c|c|c|c|c|c|}
\hline Subgroup & $\begin{array}{c}\text { Salvianolate } \\
(n=262)\end{array}$ & $\begin{array}{l}\text { Placebo } \\
(n=265)\end{array}$ & & $\mathbf{R R}$ & $95 \% \mathrm{CI}$ & Pvalue \\
\hline \multicolumn{7}{|c|}{ no. of patients/total no. (\%) } \\
\hline $\begin{array}{l}\text { Overall } \\
\text { Sex }\end{array}$ & $189 / 262(72.1)$ & $165 / 265(62.3)$ & $1 \rightarrow-1$ & 1.2653 & {$[1.0362,1.5450]$} & 0.0158 \\
\hline Male & $156 / 219(71.2)$ & $134 / 217(61.8)$ & in & 1.1535 & {$[1.0085,1.3190]$} & 0.0359 \\
\hline $\begin{array}{l}\text { Female } \\
\text { Age }\end{array}$ & $33 / 43(76.7)$ & $31 / 48(64.6)$ & - & 1.1883 & {$[0.9104,1.5510]$} & 0.2048 \\
\hline$<75$ & $157 / 221(71.0)$ & $141 / 226(62.4)$ & - & 1.1387 & {$[0.9982,1.2989]$} & 0.0524 \\
\hline$\geqslant 75$ & $32 / 41(78.0)$ & $24 / 39(61.5)$ & $\longmapsto$ & 1.2683 & {$[0.9429,1.7060]$} & 0.1072 \\
\hline \multicolumn{7}{|l|}{ Culprit vessel } \\
\hline LAD & $114 / 134(85.1)$ & $96 / 133(72.2)$ & H & 1.1786 & {$[1.0379,1.3384]$} & 0.0101 \\
\hline Non-LAD & $75 / 128(58.6)$ & $69 / 132(52.3)$ & 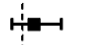 & 1.1209 & {$[0.9008,1.3948]$} & 0.3053 \\
\hline \multicolumn{7}{|l|}{ Diabetes } \\
\hline Yes & $47 / 66(71.2)$ & $43 / 61(70.5)$ & & 1.0102 & {$[0.8080,1.2630]$} & 0.9289 \\
\hline No & $142 / 196(72.4)$ & $122 / 204(59.8)$ & : & 1.2114 & {$[1.0513,1.3960]$} & 0.0076 \\
\hline $\begin{array}{l}\text { Hypertension } \\
\text { Yes }\end{array}$ & $89 / 131(67.9)$ & $97 / 143(67.8)$ & $H$ & 1.0016 & {$[0.8509,1.1789]$} & 0.9849 \\
\hline No & $100 / 131(76.3)$ & $68 / 122(55.7)$ & in-1 & 1.3696 & {$[1.1386,1.6473]$} & $<0.001$ \\
\hline
\end{tabular}

Figure 3 Prespecifed subgroup analyses of the primary endpoint between the two treatment groups. Statistical significance was determined with the Pearson's chi-square test. LAD, left anterior descending artery; RR, risk ratio; CI, confidence interval. 

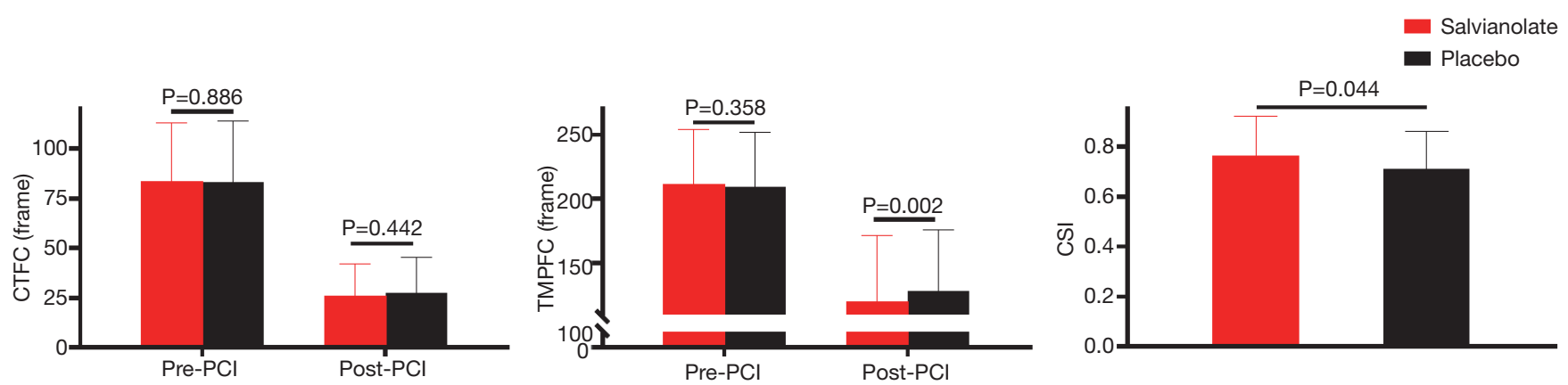

Figure 4 Continuous measurements of epicardial and myocardial perfusion ( $\mathrm{n}=220$ in the salvianolate group, $\mathrm{n}=218$ in the placebo group) and myocardial contrast echocardiography assessment ( $\mathrm{n}=60$ in the salvianolate group, $\mathrm{n}=57$ in the placebo group) in the two treatment groups Statistical significance was determined with the Student's $t$-test. CTFC, corrected TIMI frame count; TMPFC, TIMI myocardial perfusion frame count; CSI, contrast score index.

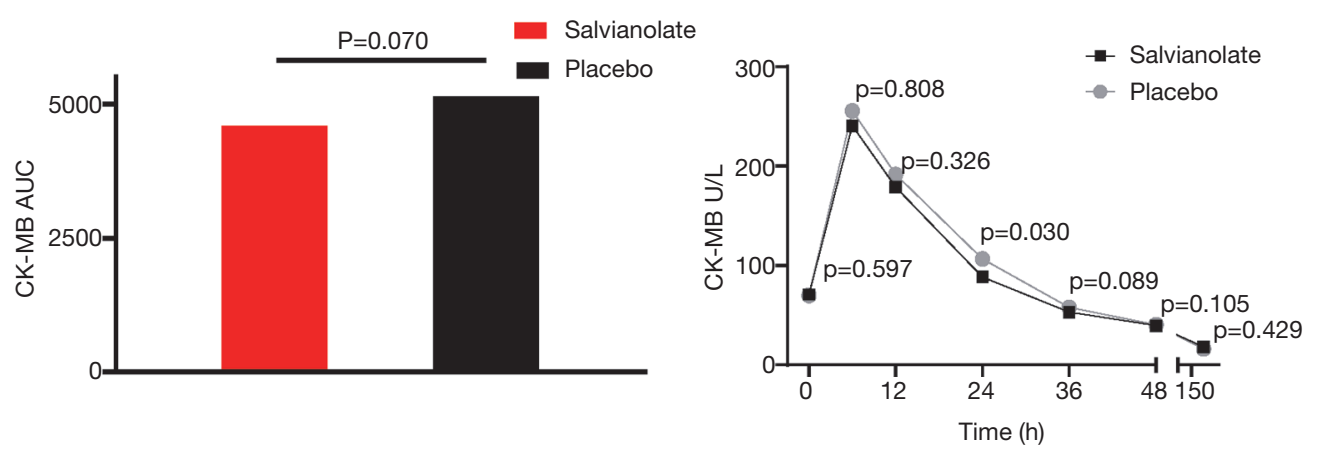

Figure 5 Comparative chronological release, represented by the AUC of CK-MB in the salvianolate (n=201) and the placebo groups (n=212). Statistical significance was determined with the Student's $t$-test. CK-MB, creatine kinase mass band fraction; AUC, area under the curve.

the difference for CTFC was not significant (Figure 4). Furthermore, in 7 days after primary PCI, these patients in the salvianolate group had higher CSI scores than the patients in the other group, which also indicated that the myocardial perfusion in the salvianolate group was considerably more improved than that in the placebo group (0.76 \pm 0.16 vs. $0.71 \pm 0.15, \mathrm{P}=0.044$; Figure 4).

\section{Enzymatic infarct size and left ventricular function}

We evaluated infarct size according to the AUC of CK-MB release. CK-MB data for sufficient data points to create a $0-36$ hour AUC were available in 362 patients (181 in the salvianolate group, 181 in the placebo group). Reasons for incomplete biomarker data included participants who died within 72 hours and patients who declined to have samples taken. There was a trend of lower CK-MB AUC in patients with salvianolate treatment, but the difference in CK-MB AUC between the salvianolate and the placebo groups was not obvious $(4,594.2 \pm 2,650.7$ vs. $5,134.4 \pm 3,109.6, \mathrm{P}=0.070)$. The CK-MB level did not differ markedly between the two groups at baseline or various time points, with the exception of 24 hours following PCI $(88.4 \pm 51.1$ vs. $106.5 \pm 76.7$, $\mathrm{P}=0.030$; Figure 5).

Echocardiography was performed to evaluate left ventricular (LV) function. Echocardiogram-assessed LVEF and LV end diastolic diameter (LVEDD) did not vary significantly between groups at the in-hospital assessment (LVEF: $57.71 \% \pm 9.64 \%$ vs. $56.36 \% \pm 9.93 \%, \mathrm{P}=0.138$; LVEDD: $48.55 \pm 4.68$ vs. $49.13 \pm 5.10 \mathrm{~mm}, \mathrm{P}=0.249$ ) or the 30 -day follow-up assessment (LVEF: $57.99 \% \pm 8.41 \%$ vs. $57.19 \% \pm 9.74 \%, \mathrm{P}=0.389$; LVEDD: $49.35 \pm 5.24$ vs. $50.23 \pm 5.77 \mathrm{~mm}, \mathrm{P}=0.238$; Figure 6). Analysis of plasma $\mathrm{N}$-terminal pro-B-type natriuretic peptide levels was 

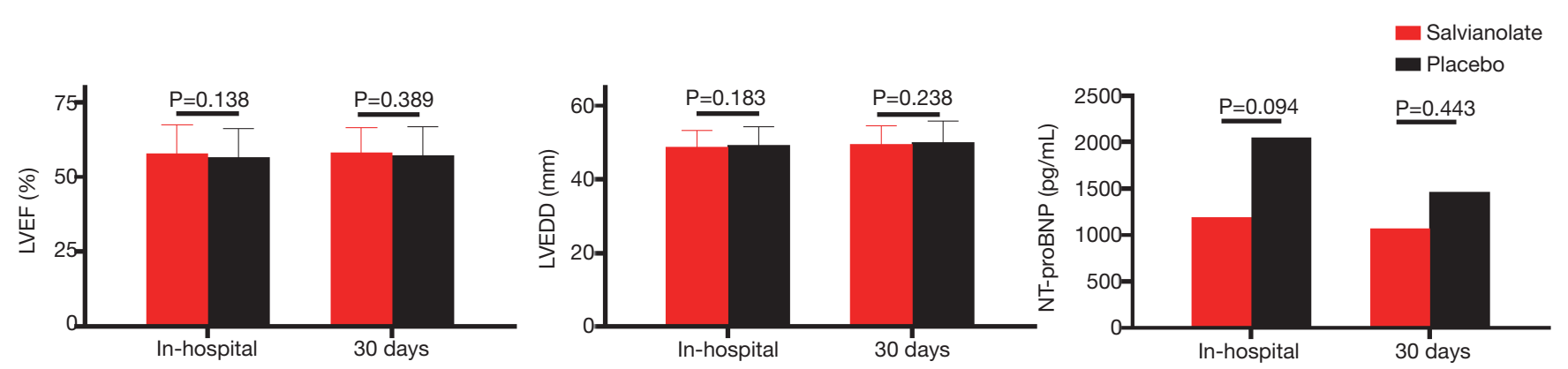

Figure 6 Left ventricular function in the two treatment arms ( $\mathrm{n}=177$ in the salvianolate group, $\mathrm{n}=182$ in the placebo group). Statistical significance was determined with the Student's $t$-test. LVEF, left ventricular eject fraction; LVEDD, left ventricular end diastolic diameter; NT-proBNP, N-terminal pro-B-type natriuretic peptide.

Table 3 Clinical outcomes and safety

\begin{tabular}{|c|c|c|c|}
\hline Variable & Salvianolate $(n=262)$ & Placebo $(n=265)$ & $P$ value \\
\hline All-cause death, n (\%) & $5(1.9)$ & $7(2.6)$ & 0.5727 \\
\hline Recurrent MI, n (\%) & $0(0)$ & $4(1.5)$ & 0.1236 \\
\hline Revascularization, $\mathrm{n}(\%)$ & $0(0)$ & $1(0.4)$ & 1 \\
\hline AEs, n (\%) & $102(38.5)^{*}$ & $113(41.7)^{\#}$ & 0.4488 \\
\hline SAEs & $14(5.3)^{\star}$ & $10(3.7)^{\#}$ & 0.3726 \\
\hline Discontinuations due to AEs & $4(1.5)^{\star}$ & $6(2.2)^{\#}$ & 0.5467 \\
\hline AEs related to study conditions & $6(2.3)^{\star}$ & $8(3.0)^{\#}$ & 0.6176 \\
\hline
\end{tabular}

*, $n=265 ;$;,$n=271$. MACEs, major adverse cardiovascular events; AEs, adverse events; SAEs, serious adverse events.

consistent with these data, with no significant difference between the two groups either in-hospital or at the 30-day follow-up.

\section{Clinical outcomes and safety}

Table 3 shows all clinical outcomes and safety parameters. The occurrence of major clinical events, including death, recurrent myocardial infarction, urgent target vessel revascularization, and stroke, were comparable between the two groups. There were five deaths in the salvianolate group and seven deaths in the placebo group $(\mathrm{P}=0.573)$. The rates of revascularization and stroke were also similar between the groups, with a trend of decreased reinfarction, but the changes were not significantly different. Regarding safety, the rate of $\mathrm{AEs}(38.5 \%$ vs. $41.7 \%, \mathrm{P}=0.449)$ ), SAEs (5.3\% vs. $3.7 \%, \mathrm{P}=0.373)$, and discontinuation due to $\mathrm{AEs}(1.5 \%$ vs. $2.2 \%, \mathrm{P}=0.547$ ) did not differ significantly between the two groups. According to investigators, six and eight events in the salvianolate group and placebo group, respectively, occurred that were related to the study medication and not to the consequence of underlying factors linked to cardiovascular disorders or the consequences of myocardial infarction.

\section{Discussion}

In this prospective randomized study, we investigated whether salvianolate administration could improve microcirculation and reduce the size of myocardial infarction in STEMI patients undergoing primary PCI. The major finding of our study is that salvianolate administration exerted beneficial effects on coronary microcirculation, although this did not significantly reduce myocardial 
infarct size as evaluated by CK-MB AUC compared with the placebo control group. During the longer term followup, the LV function measured with echocardiogram and the clinical outcomes were similar between the two groups. To our knowledge, this is the first prospective randomized study to evaluate the therapeutic effect of salvianolate in the prevention and attenuation of microvascular injury in STEMI patients undergoing primary PCI.

Despite successful restoration of epicardial coronary blood flow, a significant proportion of patients with STEMI suffer from an impairment of microvascular perfusion, which is associated with worse clinical outcomes. With respect to preventive and treatment strategies, however, no therapeutic approach has proven definitive efficacy. Microvascular injury has been considered to be the pathophysiological basis of poor myocardial perfusion in STEMI (19). Although the mechanism of microvascular injury is quite complex, ischemia-reperfusion injury and distal embolization are reported as the two major contributory factors $(20,21)$. Oxidative stress and inflammation play a pivotal role in ischemia-reperfusion injury, and a high thrombus burden is a major predictor for distal embolization. As the main active component of the Chinese medicinal material, salvia, salvianolate has been demonstrated to possess anti-oxidant and anti-inflammation activities and prevent thrombus formation through anti-platelet aggregation. In addition, some animal studies have indicated the beneficial effects of salvianolate on microvascular flow after myocardial infarction. Furthermore, the therapy of salvianolate has been reported to promote cardiac function recovery and enhance myocardial perfusion volume in patients with myocardial infarction through alleviating oxidative stress reactions and inflammatory responses (11). Here, we demonstrated for the first time in humans that not only the frequency of TMPG3 but also the frequency of complete STR and the value of TMPFC were significantly higher in patients who received salvianolate peri-operatively. These results support the notion that salvianolate effectively contributes to the improvement of myocardial perfusion after primary PCI.

As mentioned, myocardial perfusion is significantly associated with infarct size and LV function. Several animal studies have reported that salvianolate administration dramatically reduces infarct size and restored ventricular function $(14,22)$. There is evidence that in addition to improving microcirculation, salvianolate can exert direct cardioprotective effects through anti-oxidant and anti- inflammatory mechanisms. Therefore, in our study, salvianolate treatment lasted for 7 days after primary PCI, which was hoped to bring about further decrease in infarct size. In the present study, we found that there was a trend towards lower infarct size in salvianolate group, although salvianolate treatment around primary PCI did not prove significantly beneficial in attenuating myocardial damage. The reasons for this may be multifactorial. In this study, infarct size was evaluated as CK-MB AUC, which is known as an insufficiently sensitive method to detect small improvement in myocardial damage between the groups (23). Moreover, the sample size of our study, calculated based on the primary endpoint, was not powerful enough to detect a significant difference in infarct size. Furthermore, although many cardioprotective strategies have been devised and tested, early reperfusion remains the most effective method to reduce infarct size and improve ventricular function $(24,25)$. However, the mean time from the onset of symptoms to randomization was more than 5 hours for both groups in our study. As demonstrated by previous studies, the wavefront phenomenon of ischemia within 3-4 hours of occlusion of the infarct-related artery causes irreversible necrosis in the infarct-related artery supplying the area, making it almost impossible to obtain a significant reduction in the size of necrosis after that time (26). It would therefore be logical to further explore the potential cardioprotective effects of salvianolate in a new study focusing only on STEMI of a short duration ( $<4$ hours from symptom onset to PCI).

Several limitations should be considered in the interpretation of the study findings. First, salvianolate/ placebo administration was started after the patients arrived at the catheter laboratory but not at the emergency room, which might have limited the potential benefit of salvianolate. Second, we must acknowledge that the study is underpowered for clinical events and safety, and a larger clinical outcome study is needed for confirmation of our results. Third, the primary outcome of our study was recorded immediately after primary PCI, and only 1-month clinical events were evaluated. Assessment of long-term results regarding ventricular remodeling and long-term clinical outcomes is still needed.

In conclusion, this study demonstrates an improvement in myocardial perfusion when salvianolate is administered to patients with acute STEMI who are undergoing primary PCI. These encouraging results will lead to the design of a larger follow-up investigation of this intervention powered 
for clinical end points.

\section{Acknowledgments}

Funding: This study was supported by funding from the Shanghai Municipal Science and Technology Commission (No. 15DZ1900300).

\section{Footnote}

Reporting Checklist: The authors have completed the CONSORT reporting checklist. Available at http://dx.doi. org/10.21037/atm-20-6081

Data Sharing Statement: Available at http://dx.doi. org/10.21037/atm-20-6081

Conflicts of Interest: All authors have completed the ICMJE uniform disclosure form (available at http://dx.doi. org/10.21037/atm-20-6081). RX reports grants from Grant from the Shanghai Green Valley Pharmaceutical Co., outside the submitted work. The other authors have no conflicts of interest to declare.

Ethical Statement: The authors are accountable for all aspects of the work in ensuring that questions related to the accuracy or integrity of any part of the work are appropriately investigated and resolved. The study protocol was approved by the local ethics committee, and informed consent was obtained from all patients. All procedures performed in this study involving human participants were in accordance with the Declaration of Helsinki (as revised in 2013).

Open Access Statement: This is an Open Access article distributed in accordance with the Creative Commons Attribution-NonCommercial-NoDerivs 4.0 International License (CC BY-NC-ND 4.0), which permits the noncommercial replication and distribution of the article with the strict proviso that no changes or edits are made and the original work is properly cited (including links to both the formal publication through the relevant DOI and the license). See: https://creativecommons.org/licenses/by-nc-nd/4.0/.

\section{References}

1. Levine GN, Bates ER, Blankenship JC, et al. 2015 ACC/ AHA/SCAI Focused Update on Primary Percutaneous
Coronary Intervention for Patients With ST-Elevation Myocardial Infarction: An Update of the 2011 ACCF/ AHA/SCAI Guideline for Percutaneous Coronary Intervention and the 2013 ACCF/AHA Guideline for the Management of ST-Elevation Myocardial Infarction: A Report of the American College of Cardiology/American Heart Association Task Force on Clinical Practice Guidelines and the Society for Cardiovascular Angiography and Interventions. Circulation 2016;133:1135-47.

2. Lassen JF, Botker HE, Terkelsen CJ. Timely and optimal treatment of patients with STEMI. Nat Rev Cardiol 2013;10:41-8.

3. Jernberg T, Johanson P, Held C, et al. Association between adoption of evidence-based treatment and survival for patients with ST-elevation myocardial infarction. JAMA 2011;305:1677-84.

4. Jaffe R, Charron T, Puley G, et al. Microvascular obstruction and the no-reflow phenomenon after percutaneous coronary intervention. Circulation 2008;117:3152-6.

5. van Kranenburg M, Magro M, Thiele $\mathrm{H}$, et al. Prognostic value of microvascular obstruction and infarct size, as measured by CMR in STEMI patients. JACC Cardiovasc Imaging 2014;7:930-9.

6. Niccoli G, Burzotta F, Galiuto L, et al. Myocardial noreflow in humans. J Am Coll Cardiol 2009;54:281-92.

7. Hillegass WB, Dean NA, Liao L, et al. Treatment of no-reflow and impaired flow with the nitric oxide donor nitroprusside following percutaneous coronary interventions: initial human clinical experience. J Am Coll Cardiol 2001;37:1335-43.

8. Gwag HB, Kim EK, Park TK, et al. Cardioprotective Effects of Intracoronary Morphine in ST-Segment Elevation Myocardial Infarction Patients Undergoing Primary Percutaneous Coronary Intervention: A Prospective, Randomized Trial. J Am Heart Assoc 2017;6.

9. Jolly SS, Cairns JA, Yusuf S, et al. Randomized trial of primary PCI with or without routine manual thrombectomy. N Engl J Med 2015;372:1389-98.

10. Li X, Yu C, Sun W, et al. Simultaneous determination of magnesium lithospermate $\mathrm{B}$, rosmarinic acid, and lithospermic acid in beagle dog serum by liquid chromatography/tandem mass spectrometry. Rapid Commun Mass Spectrom 2004;18:2878-82.

11. Zhang Q, Jiao F, Hua C. Perioperative application of salvianolate on oxidative stress and plasma IMD/ADM2 in patients with acute myocardial infarction undergoing PCI. Exp Ther Med 2017;13:1475-9. 
12. Fei AH, Cao Q, Chen SY, et al. Salvianolate inhibits reactive oxygen species production in $\mathrm{H}(2) \mathrm{O}(2)$-treated mouse cardiomyocytes in vitro via the TGFbeta pathway. Acta Pharmacol Sin 2013;34:496-500.

13. Huang J, Yuan M, Ma J, et al. Protective Effects of Salvianolate on Contrast-Induced Nephropathy after Primary Percutaneous Coronary Intervention: A Prospective Multicenter Randomized Controlled Trial. Cardiology 2017;138:169-78.

14. Han B, Zhang X, Zhang Q, et al. Protective effects of salvianolate on microvascular flow in a porcine model of myocardial ischaemia and reperfusion. Arch Cardiovasc Dis 2011;104:313-24.

15. Ibanez B, James S, Agewall S, et al. 2017 ESC Guidelines for the management of acute myocardial infarction in patients presenting with ST-segment elevation: The Task Force for the management of acute myocardial infarction in patients presenting with ST-segment elevation of the European Society of Cardiology (ESC). Eur Heart J 2018;39:119-77.

16. Ding S, Pu J, Qiao ZQ, et al. TIMI myocardial perfusion frame count: a new method to assess myocardial perfusion and its predictive value for short-term prognosis. Catheter Cardiovasc Interv 2010;75:722-32.

17. Yang L, Xia C, Mu Y, et al. Prognostic Value of Real Time Myocardial Contrast Echocardiography after Percutaneous Coronary Intervention. Echocardiography 2016;33:421-30.

18. Van't Hof AW, Ten Berg J, Heestermans T, et al. Prehospital initiation of tirofiban in patients with STelevation myocardial infarction undergoing primary angioplasty (On-TIME 2): a multicentre, double-blind, randomised controlled trial. Lancet 2008;372:537-46.

Cite this article as: Shen L, Xu R, Yin J, Lu H, Zhang S, Huang Z, Huang D, Liu H, Qian J, Ge J; on behalf of the STEMI investigators group. Effects of salvianolate on myocardial perfusion after primary percutaneous catheter intervention in patients with ST-segment elevation myocardial infarction: a multicenter, randomized, double-blind, placebocontrolled study. Ann Transl Med 2020;8(18):1185. doi: 10.21037/atm-20-6081
19. Reinstadler SJ, Stiermaier T, Fuernau G, et al. The challenges and impact of microvascular injury in STelevation myocardial infarction. Expert Rev Cardiovasc Ther 2016;14:431-43.

20. Matsumura K, Jeremy RW, Schaper J, et al. Progression of myocardial necrosis during reperfusion of ischemic myocardium. Circulation 1998;97:795-804.

21. Gottlieb RA, Burleson KO, Kloner RA, et al. Reperfusion injury induces apoptosis in rabbit cardiomyocytes. J Clin Invest 1994;94:1621-8.

22. Qi JY, Yu J, Huang DH, et al. Salvianolate reduces murine myocardial ischemia and reperfusion injury via ERK1/2 signaling pathways in vivo. Chin J Integr Med 2017;23:40-7.

23. Pasupathy S, Tavella R, Grover S, et al. Early Use of $\mathrm{N}$-acetylcysteine With Nitrate Therapy in Patients Undergoing Primary Percutaneous Coronary Intervention for ST-Segment-Elevation Myocardial Infarction Reduces Myocardial Infarct Size (the NACIAM Trial [N-acetylcysteine in Acute Myocardial Infarction]). Circulation 2017;136:894-903.

24. Lefer DJ, Marban E. Is Cardioprotection Dead? Circulation 2017;136:98-109.

25. Hausenloy DJ, Yellon DM. Ischaemic conditioning and reperfusion injury. Nat Rev Cardiol 2016;13:193-209.

26. Reimer KA, Jennings RB. The "wavefront phenomenon" of myocardial ischemic cell death. II. Transmural progression of necrosis within the framework of ischemic bed size (myocardium at risk) and collateral flow. Lab Invest 1979;40:633-44.

(English Language Editor: J. Gray) 

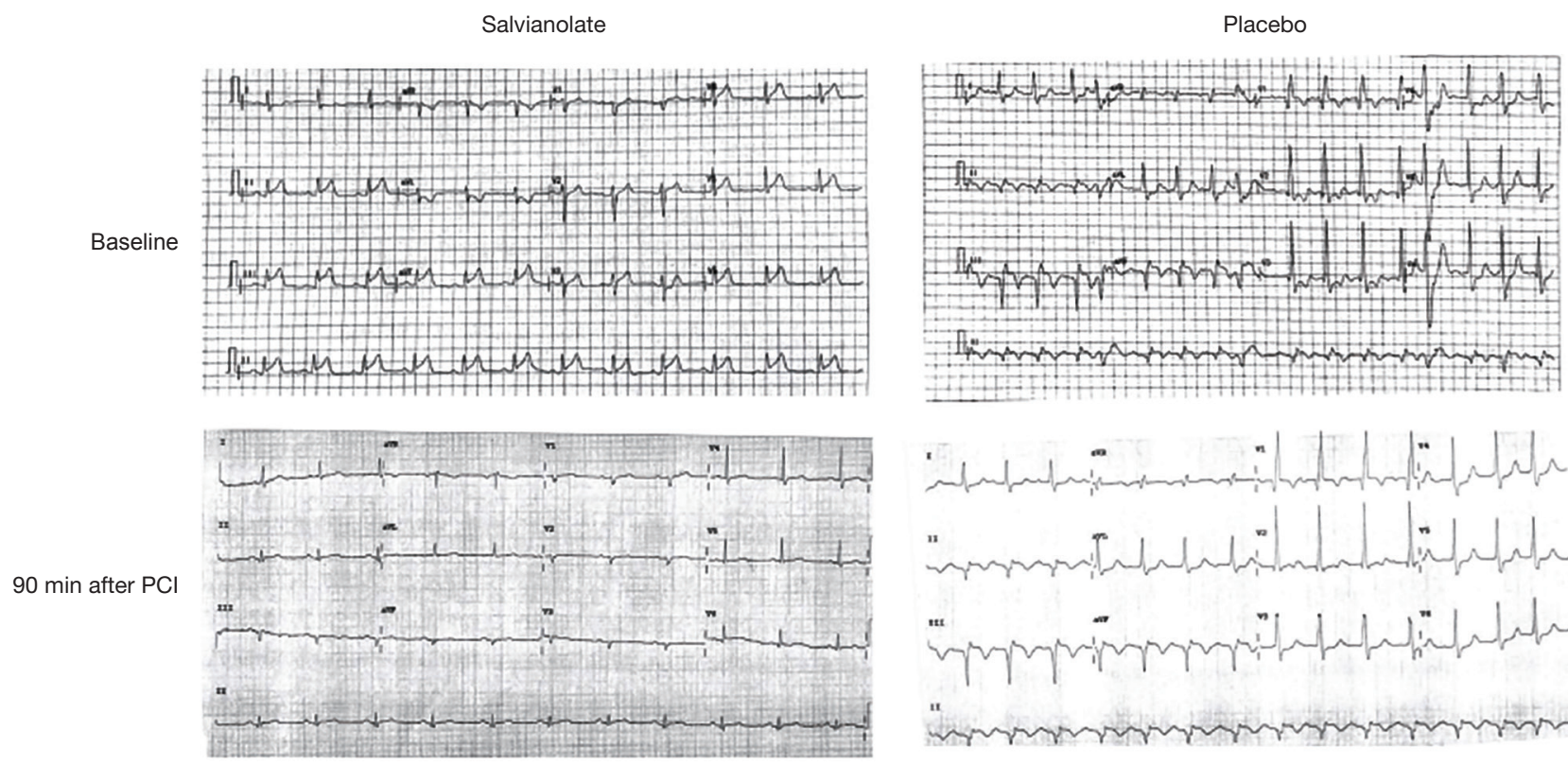

Figure S1 Representative images of electrocardiogram.

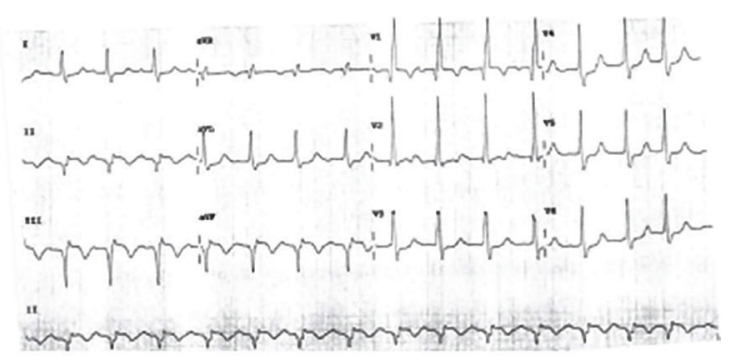

